\title{
Development and Evaluation of the Core Competencies for Master of Public Health Students
}

\author{
Y.J.Qin, G.Q. Huang, K.Zhao \& Y.G.Wang* \\ Third Military Medical University, Chongqing, 400038, China \\ *wygui@sina.com
}

\begin{abstract}
Master of Public Health (MPH) programs have been developed across the world as a response to the need for adequately trained individuals to work in the public health areas. Educational departments that deliver MPH programs have a responsibility to ensure that graduates of their programs have the basic knowledge, skills and attitudes to begin a successful career in public health. This article are concentrating on the five core competitive areas of MPH and their requirements, including Biostatistics, Environmental Health Sciences, Epidemiology, Health Policy and Management, and Social and Behavioral Sciences. Our study will provide students with a real-world public health experiences, provide students the opportunity to engage in practice-based learning, applying theoretical knowledge to practice, and should be considered for applying within MPH programs across China.
\end{abstract}

KEYWORD: Master of Public Health (MPH); Core competencies; Evaluation

\section{INTRODUCTION}

The need for change in educational practices across the health professions has been widely addressed in the literature, as well as in other seminal Institute of Medicine reports published during the past years. As a result, a resounding call for reform in health professions education, training, and professional development programs has been made in relation to curricular content, outcomes, and process (Calhoun et al. 2008). Competence is the skill to develop knowledge and ability that enhances professional practice in multiple ways. A clear and consistent definition of competency is an important step, and determination of what point to measure competency is needed (Scott-Tilley, 2008). Most importantly, competence indicators of MPH students can be used in curriculum development, planning, and learning outcome evaluation. Therefore, core competencies have been used to redefine curricula across the major health professions in recent decades. In 2006, the Association of Schools of Public Health identified core competencies for the master of public health degree in graduate schools and programs of public health in the United States.

In order to meet the needs of rapid development in public health, improve the overall quality of the public health workforces, recruiting part-time Master of Public Health for serving officers was beginning in 2001 in China. In 2009, to further adapt to the development of public health undertakings, China launched a professional degree comprehensive reform pilot work in public health education, and Peking University and Fudan University therefore were chosen as the first pilot for fresh graduates enrolled full-time master's degree in public health. In 2011 pilot units were increased to 36 universities and research institutes to train full-time master's degree in public health. Compared with part-time Master of Public Health, the purpose and mission of full-time MPH is to meet the needs of public health and international development in medical education, to further improve the public health system of personnel training, to accelerate our process in public health professionals, and to achieve convergence with the international public health graduate education. The ability to develop a sound core competency is a required skill for individuals entering the public health sector, and will ensure students are able to maximize outcomes given tight and limited resources in China.

\section{SUBJECTS AND METHODS}

\subsection{Aim}

The aim of this study was to develop the core competency indexes to measure learning outcomes of MPH students and to test its application properties.

\subsection{Design}

Based on the results of Calhoun et al. (2008), a 
cross-sectional survey design was used and the Delphi method for expert consultation was adopted.

\subsection{Sample objectives}

The inclusion criteria for consultation experts are followed as: (1) they have more than five years of work experience and education in the field of public health, specifically responsible for graduate education management and evaluation work in higher education or qualified experts in practice; (2) be familiar with the issues in this study; (3) during the consultation process, they can guarantee continued to participate in this study subjects.

According to the experts inclusion criteria, taking into account the proportion of the regional distribution and various expert, 20 experts were selected throughout the nation. They are coming from selected universities, research institutions, Health Bureaus, Centers for Disease Control and Prevention, hospitals, which are related in education of full-time Master of Public Health.

\subsection{Methods}

Delphi method was selected to conduct a questionnaire survey. Before the survey, experts were communicated by telephone or e-mail, and the issuing questionnaires were also reclaimed using email or in person. Linket-type scale was used to score the importance of indicators, which were screened by calculating the arithmetic mean, out ratio and variation coefficient index. Screening criteria are asked as follows: the importance of mean assignment indicators $>3.5$, out ratio $>0.20$, the coefficient of variation $<0.25$ (Guan \& Li, 2000). The degrees of authority are represented with an expert authority coefficient $(\mathrm{Cr})$, which is generally determined by two factors, namely, expert judgment standards for indicators (Ca) and degrees of familiarity factor (Cs), and the formula is calculated as: $\mathrm{Cr}=(\mathrm{Ca}+\mathrm{Cs}) / 2(\mathrm{Li} \&$ Shang, 2009).

\subsection{Data analysis}

SPSS 13.0 statistical software was used for analysis of collected raw data. Descriptive analysis was represented by constituent ratio and the rate; expert positive factor represented by the response rate; concentration degree of expert advice was assigned by the mean importance indicators, out ratio, and coefficient of variation; coordination level of expert advice was represented by the coefficient of variation (Qin et al. 2012).

\section{RESULTS AND ANALYSIS}

\subsection{Participants' demographics}

In this study, experts actively participated in and completed two cycles of advisory accounts for $80 \%$, with a total of 16, in which 6 people younger than 40 years old, 7 people between 40 and 50 years of age, and 3 people older than 50 years old. The majority of participants $(50.0 \%)$ come from universities, while a small number $(18.75 \%)$ are from research institute. Among them, 3 Bachelors, 8 Masters, and 5 Drs. are included; working experience for 11 to 20 years four people and more than 20 years four people; profession title: 3 professors and 4 assistant professors; professional areas: MPH education 9 people, practice application 2, administrators 2, and public health skills evaluation 1 people; tutor experience: within three years of 2 people, four to 10 years of 5 people, more than ten years, 2 people.

\subsection{The degree of expert Participation}

Expert positive coefficient means the levels of expert cares about the study and the cooperation degrees, which is reflected by the response rate of the questionnaire (Wang et al. 2010). During the first round, 18 questionnaires were returned from a total of 20 delivered questionnaires, and 18 valid questionnaires were accepted, therefore the response rate was $90.0 \%$, and the effective rate was $90.0 \%$. During the second round, 16 questionnaires were returned from a total of 18 delivered questionnaires, and 16 valid questionnaires were accepted, therefore the response rate was $88.9 \%$, and the effective rate was $88.9 \%$. The results showed that during two rounds of consultation questionnaire, expert positive coefficient> $88 \%$ was positive, indicating that selected experts are concerned about the present study, and we get a higher degree of cooperation.

The degree of expert authority: in this study the average coefficient of consultants for first and second round were 0.80 and 0.84 , respectively. It can be seen that good authority (generally $\mathrm{Cr} \geq 0.70$ ) was acceptable in this study and the consult results were credible.

Kendall coefficient of concordance (W) was used to indicate the degree of coordination of expert opinions, which reflects the degree of consistency of all expert advice for all indicators (Shi et al. 2010). The range of $\mathrm{W}$ is from $0 \sim 1$, and the larger $\mathrm{W}$ value, the better degree of coordination. During two rounds of expert studies, the $\mathrm{W}$ values were 0.392 and 0.673 , respectively, both $\mathrm{P}<0.001$, which explained that the expert advice was consistent, and coordinated well.

\subsection{Screening and modifying core capability index system}

At the first round of expert consultation questionnaire, full-time master's degree in public health system have 40 core indicators index in total, including 6 first-level indicators, and 44 secondary indicators. According to the amendments made by 
the experts and screening standards, our research group adjusted the core indicator system after discussion, deleted 10 indicators, and changed the contents of 6 indicators. We also advised that core competency systems are only restricted to the secondary indicators, but leave each university adjust third-level indicators freely according to the actual situation. During the second round of expert consultation questionnaire, 40 core competencies for MPH are advised, including 5 first-level indicators and 35 secondary indicators. The relationship between 5 first-level indicators is shown in Figure 1. Statistical results showed that mean importance scores for 40 revised indicators are between 3.68 and 4.92 , the standard deviation in the range of 0.24 to 0.79 , out ratio greater than $50 \%$, indicating all the index are in line with the screening criteria.

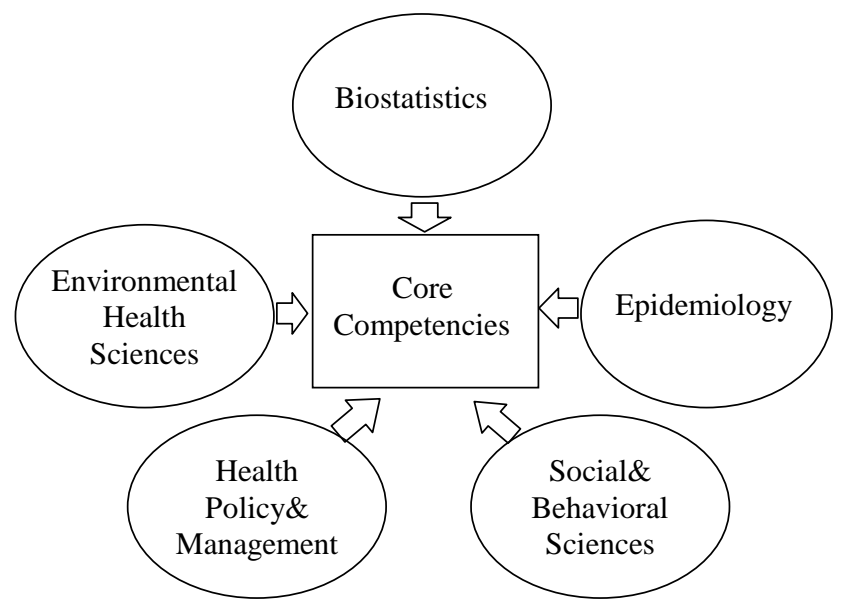

Figure 1. Master of Public Health (MPH) core competencies model.

In generally, five first-level core competency indicators tested for full-time MPH graduates are interrelated and mutually complementary. Biostatistics is the development and application of statistical methods in addressing, analyzing and solving problems in public health, biomedical, health care, clinical and population-based research. Epidemiology is the study of disease patterns and injury trends in human populations and the application of the study to the control of health problems. Environmental health science represents a study of environmental factors including physical, chemical and biological factors that affect the community health. Health policy and management is a multidisciplinary research of inquiry and practice concerned with the delivery, costs and quality of health care for individuals and populations. These definitions assume both a managerial and policy concern about the structure, process and outcomes of health services, including the financing, costs, organization, outcomes and accessibility of health care. The social and behavioral sciences in public health address the behavioral, social and cultural factors related to individual and population, and health disparities over the life course. Research and practice in these areas contribute to the development, administration and evaluation of programs and policies in public health and health services to promote and sustain healthy environments and healthy lives.

\subsection{Internal consistency of five core competencies}

Each factor and the overall scales are presented in Figure 1, and Tables also show the means, standard deviations, and the correlation coefficients between these five factors (not shown here). The means of five factors ranged from 5.37 to 6.11 , which indicated that the scholars assessed them as reasonably core competency index in MPH. The highest self-evaluation was observed in the Epidemiology (Factor 3), followed by Environmental health science (Factor 2), Biostatistics (Factor 1), and Social \& behavioral sciences (Factor 5). The lowest self-evaluated competence was identified in Health policy \& management (Factor 4). At the same time, all data indicated a high internal consistency of the items to represent the core competencies in MPH.

\section{DISCUSSION}

\subsection{Significance of building core competencies index system to $\mathrm{MPH}$}

Currently, professional graduate degree training in public health has been developed to a certain size in China, but core competencies index system and effective graduate core competency assessment tool are urgently needed. Therefore, building core competence index system of MPH, in response to the needs of society, not only provides a basis and direction for graduate training mode reform of $\mathrm{MPH}$, but also provides an assessment standard and method.

The purpose of this study was to develop a scale for assessing competencies of masters of public health and to test its properties. The conceptual framework for the competencies developed in this project contains five categories: Biostatistics, Environmental health science, Epidemiology, Health policy \& management, and Social \& behavioral sciences, and the strength of index correlations were fully in line with the practice as a multi-factorial construction. Assessing the competence of MPH students is critical to identifying areas for professional development and educational needs while ensuring that competencies are put to use for quality service. Meanwhile, our items were developed by experienced education leaders and were based largely on the conceptual framework contained in the model of the public health practice. It had been reported that using structured scoring guidelines can appropriately assess whether MPH residents have acquired core competency during training process. 


\subsection{Science significance and reliability of core competencies index system}

In this study, Delphi method was selected to build core competencies index system of MPH, which is a more recognized and more effective research tool in social science field, and ensure the authenticity of the results. When Delphi method was used in the process, the right selection of experts is the key to research success. When experts were selected in this program, distribution area, professional fields, education, job title, work experience, and other factors were fully considered. Therefore, the selected experts have a good representation of regions and disciplines, and own higher-level knowledge structure. In addition, they are interesting in this research, have higher cooperation desire and authoritative degree in this field, and we also get consistent advice in response to the data. It can be seen that the core competencies index system established in this study has a strong scientific value and high credibility.

Competency is defined as a complex combination of knowledge, skills, and abilities demonstrated by organization members that is critical to the effective and efficient function of the organization. This article discusses the MPH core competencies, which are complementary to but distinct from the specialist competencies that students should develop in their major field. The authors emphasize the importance of ongoing refinement of the core competency model with participation from both public health academics and practitioners. A competency model for MPH education is likely to produce similar benefits in terms of the attainment of skills and knowledge for effective practice. We believe our experience, this literature review, and the guiding principles provided can assist in the development of competency-based curricula.

\subsection{Limitations of this study}

Though certain success has been made in this paper, but due to limited manpower and material resources, this study also has some limitations, and it remains to be verified if the established core competencies index system can cover the knowledge, skills and attitude that a MPH student must possess, and if the evaluation systems are operability, reliability and validity. Therefore, we need continue to collect the feedback information from tutors, teachers, leaders, practitioners, and even students, modify and expand the core indicator system through simultaneous practice improvement, so that it can be more scientific and accurate.

Despite the above mentioned challenges and limitations, educators should focus on the practical value of proposed educational changes. In the next steps, as an educational community, we must agree upon a basic model of teaching and assessing each competency that also provides room for divergence and innovation. Keeping in mind the "gold standard" of improved public health, we must move towards hard outcome measures of competency.

\section{CONCLUSIONS}

Competency is an ongoing process of initial development, maintenance of knowledge and skills, educational consultation, remediation, and redevelopment, which is also different across the globe, depending on the context of the region. This study shows that the core competency has satisfactory properties and could be a useful instrument for measuring learning outcomes of MPH student, and "Epidemiology" was also found to be the most important factor contributing to $\mathrm{MPH}$ student's competencies. The results also indicated that a learning outcome perspective focuses on the learner's ability to perform a task rather than a simple possession of the knowledge required to perform the task.

\section{ACKNOWLEDGMENT}

This project was sponsored by Chinese Society of Academic Degrees and Graduate Education (B22013Y07-042), and Chinese Medical Association (2012-XW-42).

\section{REFERENCES}

[1] Calhoun, J.G., Ramiah, K., Weist, E.M. \& Shortell, S.M. 2008. Development of a core competency model for the master of public health degree. American Journal of Public Health 98(9): 1598-1607.

[2] Guan, Y.Q. \& Li, R.X. 2000. Evaluation and practice of Graduate Medical Education. Beijing: Military Medical Science Press 126-127. (in Chinese)

[3] Li, S.H. \& Shang, L.P. 2009. A preliminary study on the core competence evaluation index of nursing graduate students. Chinese Journal of Nursing Education 6(5): 198200. (in Chinese)

[4] Qin, Y.J., Zhao, K. \& Wang, Y.G. 2012. Study on the core competencies system of post graduates majored in professional clinical medicine. Chinese Journal of Social Medicine 29(3): 164-166. (in Chinese)

[5] Scott-Tilley, D.D. 2008. Competency in nursing: a concept analysis. Journal of Continuing Education in Nursing 39(2): 58-64.

[6] Shi, J.P., Gu, A. \& Li, W.X. 2010. Application of Delphi method to build standardized training assessment index system for residency doctor. Xinjiang Medical Journal 40: 110-114. (in Chinese)

[7] Wang, D., Xing, X.H. \& Cheng, Q. 2010. Application of Delphi method in screening assessment of healthy lifestyles of college students. Chinese General Practice 13(8A): 2499-2501. (in Chinese) 